\title{
Scientific Value Of Returning An Atmospheric Sample From Mars
}

\author{
White paper submitted to NASEM Planetary Sciences Decadal Survey
}

15 July 2020

Point of contact:

Bruce Jakosky

University of Colorado at Boulder

Emailbruce.jakosky@lasp.colorado.edu

Cell phone 303-817-4955

Co-authors:

Michael Amato, NASA/GSFC

Sushil Atreya, Univ. of Michigan

David Des Marais, NASA/ARC

Paul Mahaffy, NASA/GSFC

Michael Mumma, NASA/GSFC

Margaret Tolbert, Univ. of Colorado

Brian Toon, Univ. of Colorado

Chris Webster, JPL/Caltech

Richard Zurek, JPL/Caltech 


\section{Introduction and conclusions}

The previous decadal survey, Vision and Voyages for Planetary Science in the Decade 2013-2022, recommends the return of both rock and atmosphere samples in order to understand the Martian environment. The value of return of an atmospheric sample has been considered only tangentially as studies have progressed toward implementation of returned rock cores. A returned gas sample will provide an opportunity for potentially ground-breaking scientific interpretation beyond what can be gleaned from current Earth- and Mars-based measurements, including continued analysis of Martian meteorites (due to their alteration during origin and transfer to Earth). Detailed studies of the capabilities needed to implement the atmospheric sample return have not been carried out, nor has adequate attention has been given to the scientific justification for the return of atmospheric samples.

We discuss the scientific value of atmospheric samples as well as possible implementations for returning the amounts of gas necessary to carry out scientific analysis. Specific questions addressed are:

- What can be gained from such an atmospheric sample?

- How large a sample is required?

- Could the sample be passively collected or does the sample need to be concentrated, such as through pressurization and/or through a scrubber removing a major constituent?

- Are there certain constraints on materials or methodologies that might be used during sampling and containment?

Our conclusions are:

- A returned gas sample is required in order to address key questions about Mars atmospheric origin and evolution and provide necessary context for returned geological samples

- Science return depends on implementation selected:

- Ambient-pressure sample retained in the head space in the sample-return canister: Inadequate due to (i) lack of getter to remove material outgassed from walls prior to filling with atmospheric gas, (ii) chemical interaction of gas with sample during transit to Earth

- Ambient-pressure sample container with 100 cc volume: Utilizes valves and a getter; will be adequate for most atmospheric gases, including noble gases, but not methane or ethane; significantly improved precision for some noble gases would obtain with addition of scrubber.

- Gas compressed from 7 mbar to $\sim 0.7$ bar pressure: Provides 100 times the gas sample size, allows determination of $\mathrm{CH}_{4}$ abundance to $\sim 1$ part-per-trillion and the ${ }^{13} \mathrm{C} /{ }^{12} \mathrm{C}$ isotope ratio in $\mathrm{CH}_{4}$ to $\sim 4$ per mil. This large gas sample would allow multiple allocations to state-of-art laboratories and further enrichment as necessary.

- Most-exciting science results from return of compressed sample

- Returning a sample of dust filtered out of the atmosphere is important for understanding Martian chemistry

\section{Current State of Atmospheric Trace Gas/Isotope Measurements}


Measurements of Mars atmospheric trace gas abundance and isotopic composition have been made by a number of techniques, including ground-based and Earth-orbital remote sensing of the Martian atmosphere; in situ sampling during atmospheric passage (Viking entry, MAVEN aeropasses) and after landing (Viking, Mars Science Laboratory); remote sensing from Mars orbit (e.g., Mars Express, ExoMars Trace Gas Orbiter); and analysis of atmospheric gas trapped in Martian meteorites recovered on Earth. While much has been learned, limitations in the sensitivity and coverage of the remote and in situ measurements made to date limit addressing key questions of solar-system science. Analysis of Martian meteorites can be conducted with state-of-the-art terrestrial laboratories, but interpretation is limited by the unknown provenance of the meteorites, and by their alteration both from the originating impact and due to exposure to cosmic radiation during transfer through space to Earth.

Key questions that can be addressed are:

- Atmospheric origin and climate evolution of Mars, and comparisons between processes on Mars with the other terrestrial planets Earth and Venus

- Devolatilization of an early magma ocean, crustal release, and loss to space by early hydrodynamic outflow, massive impacts, and ongoing sputtering processes all are traced through noble gas isotopes and elemental ratios

- Atmospheric source and loss processes to both space and surface sinks over time are revealed by comparison of current atmospheric elemental and isotopic composition of noble gases and light elements such as $\mathrm{H}, \mathrm{C}, \mathrm{N}$, and $\mathrm{O}$ with these same values in samples of ancient Mars returned to Earth by the Mars 2020 mission, sampled in Martian meteorites, or studied by surface landers such as MSL.

- Finally, the presence and origin of methane and other hydrocarbon species to address ongoing geological processes and the potential occurrence of extant life on Mars

\section{Scientific value of returning a sample of the Martian atmosphere}

\section{A. Xenon}

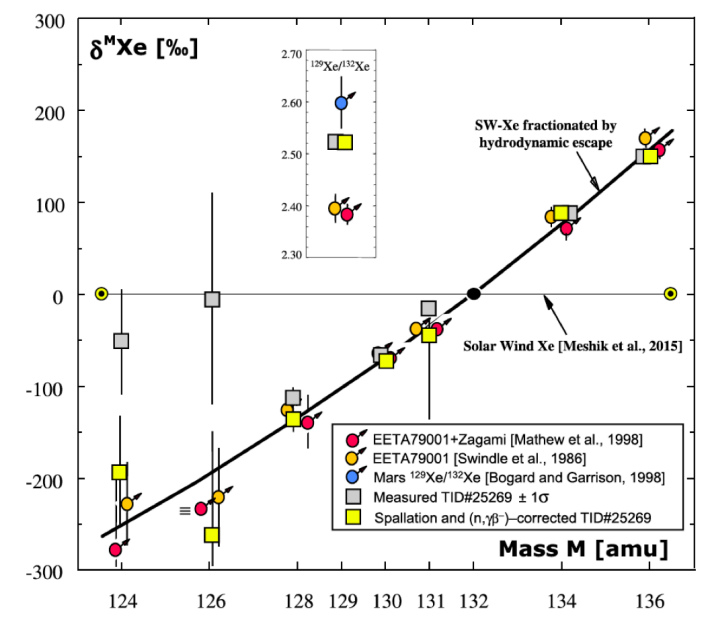

Fig. 1. Isotopic measurements of Xe made from Martian meteorites and on the surface with MSL/SAM. From Conrad et al. 2016.

Xe isotopes reflect mass fractionation of solar-like Xe via early hydrodynamic loss to space, telling us about earliest history of atmosphere fractionation (with the addition of ${ }^{129} \mathrm{Xe}$ from decay of ${ }^{129} \mathrm{I}$ ). Figure 1 shows the currently available measurements of Xe isotopes. 
Although we have measurements in Martian meteorites, the gases sampled does not reflect unadulterated atmosphere - they include a combination of atmospheric, mantle, and spallation (in space) contributions. MSL/SAM measurements disagree with the meteorite values for ${ }^{124} \mathrm{Xe}$ and ${ }^{126} \mathrm{Xe}$ isotopes; this is attributed to Ba spallation and release to the atmosphere. An atmospheric sample would yield much more precise measurements than SAM for the two trace isotopes and could resolve differences with meteorite values. Better precision might also clarify a possible minor ${ }^{244} \mathrm{Pu}$ fission component (present in Earth's atmosphere).

\section{B. Krypton}

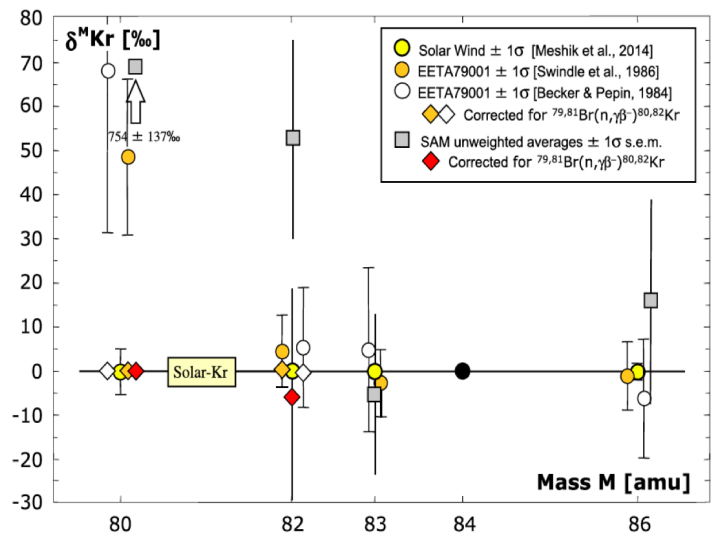

Fig. 2. Isotopic measurements of Kr made from Martian meteorites and on the surface with MSL/SAM. From Conrad et al. 2016.

Kr shows very different isotopic fractionation pattern from Xe (Figure 2). This likely tells us about efficacy of early hydrodynamic outflow and its timing relative to subsequent outgassing. Again, meteorite measurements do not provide samples of unadulterated atmosphere, which a properly sized returned air sample could do. MSL/SAM made measurements, but there are large error bars, plus unable to measure ${ }^{78} \mathrm{Kr}$ due to background gas interference.

\section{Other atmospheric isotopes}

\begin{tabular}{|l|c|c|}
\hline Isotopes & $\begin{array}{l}\text { Mars value } \\
\%\end{array}$ & $\begin{array}{l}\text { SAM Suite } \\
\text { QMS/TLS }\end{array}$ \\
\hline$\delta^{38} \mathrm{Ar}_{\text {Sun }}$ & $310 \pm 31$ & QMS \\
\hline$\delta^{40} \mathrm{Ar}_{\text {Earth }}$ & $5419 \pm 1013$ & QMS \\
\hline$\delta^{15} \mathrm{~N}_{\text {Earth }}$ & $572 \pm 82$ & QMS \\
\hline$\delta^{13} \mathrm{C}_{\text {VPDB }}$ & $45 \pm 12$ & QMS \\
\hline$\delta^{13} \mathrm{C}_{\text {VPDB }}$ & $46 \pm 4$ & TLS \\
\hline$\delta^{18} \mathrm{O}_{\text {SMOW }}$ & $48 \pm 5$ & TLS \\
\hline$\delta D_{\text {SMOW }}$ & $4950 \pm 1080$ & TLS \\
\hline
\end{tabular}

Fig. 3. Current best estimates of isotopic ratios in the Martian atmosphere. Values given in $\delta$ notation in per mil (parts per thousand), where: $\delta^{a} X=\left(\frac{{ }^{a} R_{\mathrm{x}}}{{ }^{a} R_{\text {std }}}-1\right) \times 1000$

Other atmospheric isotopes (Figure 3) are used to distinguish processes by which atmosphere has evolved, including escape to space, exchange with crustal and polar reservoirs, outgassing of juvenile gases; focus is on key gases - noble gases, $\mathrm{H}_{2} \mathrm{O}, \mathrm{CO}_{2}, \mathrm{~N}_{2}, \mathrm{O}_{2}$. Light isotopes in the geological samples returned from the ancient Jezero crater that were formed in a lake environment will be measured with high precision in terrestrial laboratories. Comparison 
with equally high-precision isotopic measurements from a sample of the current atmosphere will enable more robust models of processes that led to planetary change.

Neon isotopes were not measured by MSL because of isobaric interferences. Baines et al. (2013) suggested that generation of a terrestrial planet mass fractionation line of the ${ }^{22} \mathrm{Ne} /{ }^{20} \mathrm{Ne}$ and ${ }^{21} \mathrm{Ne} /{ }^{20} \mathrm{Ne}$ ratios would discriminate source reservoirs for Venus, Earth, and Mars.

\section{Methane and other hydrocarbons}

Methane is a strong indicator of geological and biological activity; abundance and isotopic measurements can discriminate between these mechanisms. In absence of finding organisms in returned geologic samples, such measurements could provide the strongest indicators if life is present today. Earth-based telescopic, MSL/SAM, and TGO data are potentially inconsistent; near-surface methane measurements at a new location are needed. Measurement of ${ }^{13} \mathrm{C}$ might provide strong constraints on origin mechanism. In addition, the $\mathrm{CH}_{4}$ abundance relative to $\mathrm{C}_{2} \mathrm{H}_{6}$ and $\mathrm{C}_{3} \mathrm{H}_{8}$ might provide strong constraints on origin; the combination of $\delta^{13} \mathrm{C}$ and the ratio $\mathrm{CH}_{4} /$ heavier hydrocarbon ratio will provide even stronger constraint on origin (Figure 4).

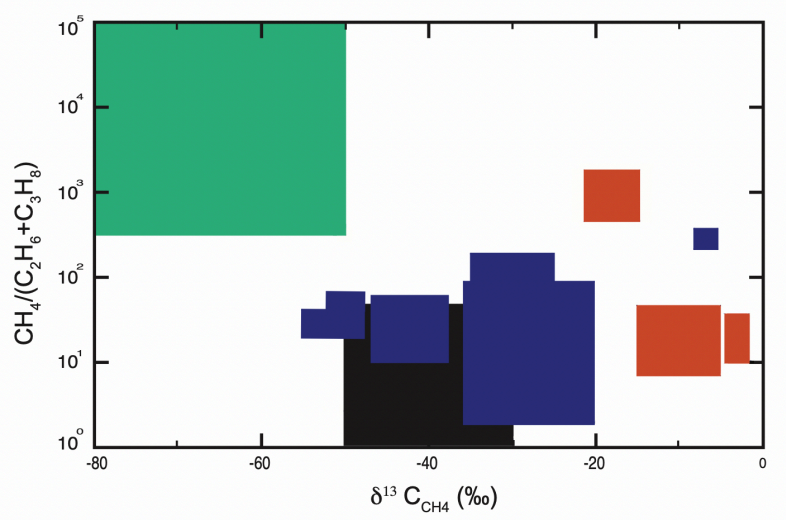

Fig. 4: Correlation of the ratio methane/(ethane + propane) with $\delta^{13} \mathrm{C}$ (methane) for various sources of terrestrial methane. Green: microbial methanogenesis due to either reduction of $\mathrm{CO}_{2}$ or fermentation; blue: Low temperature water-rock reactions; red: high temperature water-rock reactions; black: thermogenesis. Note the clear discrimination between methane produced by microbial methanogenesis versus the various abiogenic sources. (From Allen et al. 2006, modified from Horita and Berndt 1999).

\section{E. Trace gases in the air and adsorbed onto airborne dust grains}

The atmosphere of Mars is expected to contain an array of trace gases besides methane; of particular interest are $\mathrm{SO}_{2}$ and the halogens, especially $\mathrm{HCl}$. These gases can provide additional insight into the formation, source and the outgassing mechanisms of methane, as well as any current fumarolic activity on Mars. These gases have not been detected on Mars; they could be important at abundances lower than the current detection limit of $0.3 \mathrm{ppbv}$. Much lower abundances can be measured in the returned atmospheric sample at Earth.

In addition to their possible presence as gases in the Martian atmosphere, sulfur- and halogen-bearing species are expected to be present on the airborne dust. Airborne dust on Mars serves as a giant surface on which heterogeneous processes can take place suspended in the atmosphere. Besides providing a means for surface catalysis in the air, the airborne dust can trigger heterogeneous chemistry involving the adsorbed sulfur and halogen species. Returning a sample of airborne dust will enhance the scientific value of a returned atmospheric sample (in addition to the separate value of returning airborne dust for its own sake, not discussed here). 


\section{Possible implementations for returning an atmospheric sample}

Multiple configuration options exist (Figure 5); each would address sample-volume requirements differently, and all contain high TRL components. Each option would include a getter to remove residual gas outgassed from container walls during the cruise phase of the mission. The sample container would utilize low-outgassing surface-passivated metal materials to minimize contamination. Valves and valve controllers are flight-qualified devices developed for SAM, on the order of $25 \mathrm{~g}$ each; leak rates $<1 \times 10^{-9} \mathrm{~atm}$-cc/sec are sufficient to retain the gas sample robustly over many years. Valves and compresser (where utilized) are controlled by circuits that remain on Mars, minimizing the required mass of the returned material.

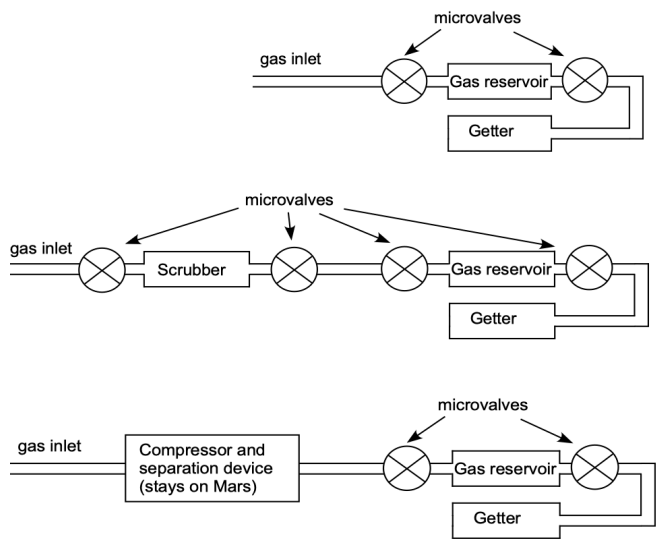

Fig. 5. Possible implementation options for returning gas sample. Option 1 (top): Ambient-pressure gas reservoir. Option 2 (middle): Ambient-pressure gas reservoir with scrubber for enrichment by $25 x$ in noble gases. Option 3 (bottom): Gas reservoir pressurized to 1 bar or more with compressor; separation device and electronics stay on Mars

In the implementation involving gas compression, existing technology could be utilized. For example, MOXIE on Mars 2020 uses an Air Squared compressor $(2.3 \mathrm{~kg}, 100 \mathrm{~W})$ designed for large gas amount, flow rates; a miniature scroll pump by Creare $(350 \mathrm{~g}, 5 \mathrm{~W})$ developed for Mars under SBIR. The compressor could be mounted on the lander and not be a part of samplecanister mass that is returned to Earth; for example, it could utilize a solenoid release/separation mechanism, with Schrader-like input valve in series with microvalve seal.

Airborne dust also could be collected with addition of 3 valves and a dust filter (Figure 6). After gas reservoir is filled and reservoir valves closed, large volumes of Mars air would be pumped through filter to collect and trap dust and its valves closed.

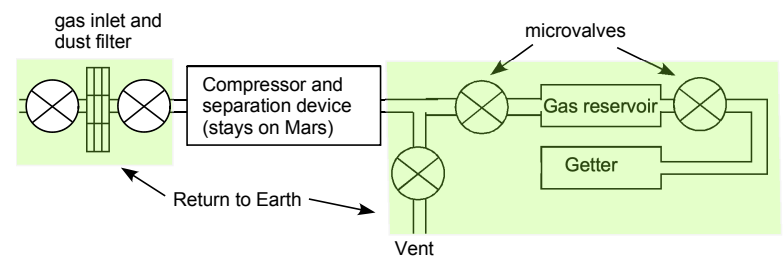

Fig. 6. Implementation that would allow filtering of large volumes of dust for return to Earth.

\section{Gas volume requirements}

The returned-gas volume (and therefore the implementation selected) depends on the science results desired. We examine two driving cases.

A. ${ }^{126} \mathrm{Xe}$ as the driving case for noble gases 
Mass spectrometer sample and analysis assumptions:

- Half of the sample to be retained for future analysis

- Remaining half provides 9 sample splits - 3 delivered to each of 3 separate labs for triplicate analysis

- $2500 \mathrm{cc}$ ion counting analyzer with $5 \times 10^{-3}(\mathrm{c} / \mathrm{sec}) /(\mathrm{part} / \mathrm{cc})$

- $100 \mathrm{sec}$ integration on each isotope

- Errors limited by counting statistics

Obtainable precision:

- Ambient-pressure reservoir with $\sim 50 \mathrm{cc}$ reservoir volume (option 1)

- $2 \times 10^{-5}$ moles of Mars gas to Earth

- 10 per mil precision

- consistent with 2011 E2E-ISAG analysis

- Scrubber plus ambient-pressure $200 \mathrm{cc}$ reservoir volume (option 2)

- $5 \times 10^{-4}$ moles of Mars gas to Earth

- 1 per mil precision

- Compression of gas not required for Noble gases

B. ${ }^{13} \mathrm{C} /{ }^{12} \mathrm{C}$ as the driving case for $\mathrm{CH}_{4}$

Assumptions:

- Assumes atmospheric sample is collected from near-surface at night with $0.5 \mathrm{ppbv}$ methane content, as measured by MSL/SAM

- 100 cc sample-return container (with or without scrubber or compression)

- Non-destructive analysis using tunable diode laser spectroscopy

Results are shown in Figure 7. The basic result is that the key isotopic measurement at useful levels of uncertainty is possible only with on-Mars compression of the returned gas sample.

\begin{tabular}{|c|c|c|c|}
\hline Method & 813C_CH4 uncertainty & Notes & Fig. 7. Analysis of \\
\hline $\begin{array}{l}\text { A. Direct ingest to } 7 \mathrm{mbar} \text { then } \\
\text { return }\end{array}$ & Not possible & & $\begin{array}{l}\text { implementation options for } \\
\text { determining }{ }^{13} \mathrm{C} /{ }^{12} \mathrm{C} \text { ratio in }\end{array}$ \\
\hline $\begin{array}{l}\text { B. Enrich } 25 x \text { with scrubber on } \\
\text { Mars then return }\end{array}$ & 400 per mil & Final pressure $\sim 5$ mbar. & methane. \\
\hline $\begin{array}{l}\text { C. Compress to } 0.7 \text { bar pressure } \\
\text { on Mars then return }\end{array}$ & 100 per mil & $\begin{array}{l}\text { Equivalent to } 100 \text { samples at } 7 \\
\text { mbar, easily divided up. }\end{array}$ & \\
\hline $\begin{array}{l}\text { D. Compress sample on Mars, } \\
\text { then enrich } 25 x \text { on Earth }\end{array}$ & 4 per mil & Many options at this stage & \\
\hline
\end{tabular}

\section{References.}

Allen, M., et al., Is Mars Alive? Eos, Trans. Amer. Geophys. Union, 87, 433-448, 2006.

Conrad, P.G. et al., In situ measurement of atmospheric krypton and xenon on Mars with Mars Science Laboratory, Earth Planet. Sci. Lett., 454:1-9, doi: 10.1016/j.epsl.2016.08.028, 2016.

Baines K. H., et al., The atmospheres of the terrestrial planets: Clues to the origins and early evolution of Venus, Earth, and Mars. In Comparative Climatology of Terrestrial Planets (S.J. 
Mackwell et al., eds.), Univ. of Arizona, Tucson, DOI: 10.2458/azu_uapress_9780816530595ch006, 2013.

Horita, J., and M. E. Berndt, Abiogenic methane formation and isotopic fractionation under hydrothermal conditions, Science, 285, 1055-1057, 1999. 